\title{
SOME INTEGRAL RELATIONS
}

\section{H. BATEMAN}

Let $u(r)$ have a continuous derivative for positive values of $r$, then a solution of the integral equation

$$
u(r)=\int_{0}^{\pi / 2} \cos (k r \sin a) U(r \cos a) d a
$$

is given by the formula

$$
U(s)=(2 / \pi)\left[u(0) \operatorname{ch}(k s)+s \int_{0}^{\pi / 2} \operatorname{ch}(k s \sin b) u^{\prime}(s \cos b) d b\right] .
$$

The mean value theorem for a two-dimensional potential round a circle, due essentially to Parseval, gives the equation

$$
1=(2 / \pi) \int_{0}^{\pi / 2} \cos (k r \sin a) \operatorname{ch}(k r \cos a) d a,
$$

and so the relation to be established is

$$
u(r)-u(0)=(2 / \pi) \int_{0}^{\pi / 2} \cos (k r \sin a) r \cos a d a \int_{0}^{\pi / 2} \operatorname{ch}(k x) u^{\prime}(y) d b
$$

where

$$
y=r \cos a \cos b, \quad x=r \cos a \sin b=z \sin c, \quad z=\left(r^{2}-y^{2}\right)^{1 / 2} .
$$

When the quantities $y$ and $c$ are taken as new variables of integration the integral on the right becomes

$$
(2 / \pi) \int_{0}^{r} u^{\prime}(y) d y \int_{0}^{\pi / 2} \cos (k z \cos c) \operatorname{ch}(k z \sin c) d c=\int_{0}^{r} u^{\prime}(y) d y
$$

and so the formula is established. ${ }^{1} \mathrm{~A}$ proof of the uniqueness of the solution is not easy to obtain by substitution of an integral of type (1) in (2) but may be derived from the relation

Received by the editors March 27, 1944.

1 The case $k=0$ is reducible to Abel's integral equation and is a special case of an equation $F(x)=\int_{0}^{\pi / 2} f(x \cos a) \sin ^{2 n+1} a d a$ found by Poisson in his Researches on heat conduction, J. Ecole Polytech. vol. 12 (1823) p. 299. This equation was discussed by J. Liouville, ibid. vol. 15 (1835) pp. 55-60. Professor M. A. Bosoco has kindly recalled my attention to the interesting integral transformation used for this case by O. Schlömilch, Zeitschrift für Mathematik und Physik vol. 2 (1857). 


$$
u\left(s^{1 / 2}\right)=\frac{1}{2} \int_{0}^{s} \cos k(s-t)^{1 / 2} U\left(t^{1 / 2}\right) d t /[t(s-t)]^{1 / 2}
$$

and the equivalent relation

$$
\int_{0}^{\infty} e^{-z s} u\left(s^{1 / 2}\right) d s=\frac{1}{2} \int_{0}^{\infty} e^{-z t} U\left(t^{1 / 2}\right) t^{-1 / 2} d t \int_{0}^{\infty} e^{-z t} \cos \left(k t^{1 / 2}\right) t^{-1 / 2} d t .
$$

Thus when $u(r)$ is given for positive values of $r$ the first integral on the right is determined uniquely and so by a simple extension of Lerch's theorem $U(r)$ is unique.

The theory of exponential integrals has been advanced by the recent tabulation ${ }^{2}$ of the function $F(x)$ which is defined by the integral

$$
\begin{aligned}
F(x) & =\int_{0}^{\infty} e^{-m^{4} / 4} \cos (m x) d m \\
& =\frac{1}{2}\left(\frac{1}{2}\right)^{1 / 2} \sum_{n=0}^{\infty}\left(-2 x^{2}\right)^{n} \Gamma(n / 2+1 / 4) /(2 n) !
\end{aligned}
$$

The table indicates that $F(x)=0$ for $x=2.435,4.778$, and 6.550 , approximately. This is in accordance with the result of $\mathrm{F}$. Bernstein ${ }^{3}$ that $F(x)$ has an infinite number of real zeros.

The asymptotic behavior of $F(x)$ for large complex values of $x$ has been discussed by Burwell ${ }^{4}$ who gives, moreover, an asymptotic formula for the large zeros. The asymptotic expansion of $F(x)$ for large positive values of $x$ is rather complicated but it is sufficient to establish the convergence of the integral

$$
\int_{0}^{\infty} e^{-z t} F\left(t^{1 / 4}\right) t^{-1 / 4} d t=\frac{\pi z^{-3 / 4}}{2} \exp \left(-z^{-1 / 2} / 4\right), \quad R(z)>0,
$$

which may be regarded as a generating function of $F\left(t^{1 / 4}\right) t^{-1 / 4}$ in the sense of Abel. In the theory of Haar and others by which the asymptotic behavior of a function for large positive values of $t$ is derived from the behavior of the generating Laplacian integral at points $z$ on the line bounding the region of convergence, the form of the function

$$
F\left(t^{1 / 4}\right) t^{-1 / 4}
$$

for large positive values of $t$ is characterized by the behavior of the

${ }^{2}$ G. Grimminger, Velocity and mass distributions resulting from the lateral diffusion of a current in a stratified medium on a rotating earth, Journal of the Franklin Institute vol. 236 (1943) pp. 413-443, 509-520.

${ }^{3}$ F. Bernstein, Math. Ann. vol. 79 (1918), pp. 265-268. See also G. P6lya, Messenger of Mathematics vol. 52 (1923) pp. 185-188.

${ }^{4}$ W. R. Burwell, Proc. London Math. Soc. (2) vol. 22 (1924) pp. 57-72. 
function on the right of (5) at the point $z=0$. The type of singularity is in this case different from that of any tabulated generating function.

The relation (5) was obtained originally by substituting the infinite series for $F(x)$ and integrating term by term..$^{5}$ An alternative plan is to consider the function

$$
U(x, y)=\int_{0}^{\infty} e^{-y m^{4}} \cos (m x) d m
$$

which is a solution of the partial differential equation

$$
U_{y}+U_{x x x x}=0 .
$$

The integral

$$
u(y, z)=\int_{0}^{\infty} e^{-z t} U\left(t^{1 / 4}, y\right) t^{-1 / 4} d t=4 \int_{0}^{\infty} e^{-z x^{4}} U(x, y) x^{2} d x
$$

is then a solution of the partial differential equation

$$
u_{y}=360 z u+2160 z^{2} u_{z}+1664 z^{3} u_{z z}+256 z^{4} u_{z z z}
$$

and may be identified with the solution

$$
u(y, z)=\frac{1}{2}\left(\frac{1}{2}\right)^{1 / 2} \pi y^{-1 / 4} z^{-3 / 4} \exp \left(-y^{-1 / 2} z^{-1 / 2} / 8\right)
$$

for $U$ is of the form $(1 / x) f\left(y x^{-4}\right)$ and so $u(y, z)$ is of the form

$$
u(y, z)=z^{-1 / 2} H(y z)=z^{-1 / 2} H(w),
$$

say. If $H(w)=w^{-1 / 4} S\left(w^{-1 / 2}\right)$ the differential equation for $S(s)$ is

$$
2 s d T / d s+T=0 \text { where } T=64 S^{\prime \prime}(s)-S .
$$

Hence $T=C s^{-1 / 2}$ and so $S=A e^{s / 8}+B e^{-s / 8}-C P(s)$, where for large values of $s$ the function $P$ has an asymptotic expansion of type

$$
P(s)=s^{-1 / 2}\left[1+1 \cdot 3\left(16 / s^{2}\right)+1 \cdot 3 \cdot 5 \cdot 7\left(16 / s^{2}\right)^{2}+\cdots\right] .
$$

The solution $S=B \exp (-s / 8)$ is of the right type when $s$ is large and $w$ small and the proposed value of the integral seems to be correct.

There are some related integrals involving the functions $V_{0}, V_{1}, V_{2}$, $V_{3}$ defined on p. 114 of my Partial differential equations of mathemati-

\footnotetext{
5 For a justification of this process reference may be made to G. H. Hardy, Transactions of the Cambridge Philosophical Society vol. 21 (1908) pp. 1-48 and E. C. Titchmarsh, The theory of functions, Oxford, 1932, p. 348. The infinite series for $F(x)$ may indeed be expressed as the difference of two series involving positive terms and each of these may be integrated term by term because the resulting series are convergent. I owe the second reference to Morgan Ward.
} 
cal physics. These functions are solutions of the partial differential equation ${ }^{6}$

$$
V_{y}=V_{x x x x}
$$

and are represented by the integrals

$$
\begin{aligned}
& V_{0}(x, y)=4 \int_{0}^{\infty} e^{-4 y s^{4}} \cos s x \operatorname{ch} s x d s, \\
& V_{2}(x, y)=4 \int_{0}^{\infty} e^{-4 y s 4} \sin s x \operatorname{sh} s x d s, \\
& V_{1}(x, y)=4 \int_{0}^{\infty} e^{-4 y s t}(\sin s x \operatorname{ch} s x+\cos s x \operatorname{ch} s x) d s, \\
& V_{3}(x, y)=4 \int_{0}^{\infty} e^{-4 y s 4}(\sin s x \operatorname{ch} s x-\cos s x s h s x) d s .
\end{aligned}
$$

The simplest of the integrals are

$$
\begin{aligned}
& \int_{0}^{\infty} e^{-z t t^{-1 / 4}} V_{0}\left(s^{1 / 4}, y\right) d t=\pi y^{-1 / 4} z^{-3 / 4} \cos \left(y^{-1 / 2} z^{-1 / 2} / 8\right), \\
& \int_{0}^{\infty} e^{-z t t^{-1 / 4}} V_{2}\left(s^{1 / 4}, y\right) d t=\pi y^{-1 / 4} z^{-3 / 4} \sin \left(y^{-1 / 2} z^{-1 / 2} / 8\right) .
\end{aligned}
$$

The asymptotic forms of $V_{0}(x, y)$ and $V_{2}(x, y)$ for large values of $x$ can be derived from Burwell's results; they can also be regarded as determined by the behavior of the functions on the right in the neighborhood of $z=0$. In the search for functions whose generating functions have prescribed singularities it should be worth while to fully explore the functions provided by solutions of the general equation of Poisson.

Generating functions depending on the error function and the integrals of Fresnel may be derived from the functions now under discussion. Thus

$$
\begin{aligned}
\int_{0}^{\infty} e^{-z t}[F(0)- & \left.F\left(t^{1 / 4}\right)\right] t^{-5 / 4} d t \\
= & \frac{\pi z^{-1 / 4}}{2} \sum_{n=0}^{\infty}\left(-z^{-1 / 4} / 4\right)^{n} /[(2 n+1)(n+1) !], \\
\int_{0}^{\infty} e^{-z t}[V(0, y) & \left.-V\left(t^{1 / 4}, y\right)\right] t^{-5 / 4} d t \\
= & \frac{\pi}{16}\left(y^{5} z^{3}\right)^{-1 / 4} \sum_{n=0}^{\infty}\left(-8 y^{1 / 2} z^{1 / 2}\right)^{-n} /[(4 n+3)(2 n+2) !],
\end{aligned}
$$

( $T$ This is a special case of the equation $\partial^{m} V / \partial x^{m}=\partial^{n} V / \partial y^{n}$ discussed by Poisson, J. Ecole Polytech. vol. 12 (1823) pp. 464-474. 


$$
\begin{aligned}
\int_{0}^{\infty} e^{-x t} V_{2}\left(t^{1 / 4}, y\right) t^{-5 / 4} d t \\
=\frac{\pi y^{-3 / 4} z^{-1 / 4}}{2} \sum_{n=0}^{\infty}\left(-8 y^{1 / 2} z^{1 / 2}\right)^{-n} /[(4 n+1)(2 n+1) !] .
\end{aligned}
$$

For the solution of the general equation of Poisson, reference may be made to H. Block, Arkiv för Matematik, Astronomi och Fysik vol. 8 (1913), G. Mikaelsson, Dissertation, Upsala, 1920 and E. Rothe, Math. Zeit. vol. 28 (1928) pp. 48-72.

California Institute of Technology 